\title{
利用芳基乙烷的脱氢硝化合成硝基芳香烯烃的新方法
}

\author{
穆 兵 ${ }^{a, b}$ 吴俊良 ${ }^{*, a} \quad$ 张广安 $^{a}$ \\ ( ${ }^{a}$ 郑州大学化学学院 郑州 450001) \\ $(b$ 郑州师范学院化学化工学院 郑州 450044)
}

\begin{abstract}
摘要 硝基烯烃是有机合成化学中常见的重要中间体，其合成方法主要通过硝基烷烃与醛或酮的缩合、消除，烯烃直 接脱氢硝化或者烯基羧酸脱羧硝化得到目标产物，但是这些合成方法由于原料价格昂贵，在大规模生产中受到限制. 本研究首次采用廉价易得的芳基乙烷与硝酸钡为原料, 以铜/银为催化剂, 过硫酸钾为氧化剂, 通过脱氢硝化反应合成 硝基芳香烯烃. 在优化的反应体系中, 1,1 -二苯基乙烷、苯基乙烷、4-乙基联苯及乙基菜类化合物能与硝酸钡进行脱氢 硝化反应，以中等至好的收率获得 $E$ 型硝基芳香烯烃.
\end{abstract}

关键词＼cjkstart硝基芳香烯烃; 芳基乙烷；硝酸钡；脱氢硝化；过硫酸铵

\section{Alternative Approach for the Synthesis of Nitroaromatic Olefins via Dehydrogenative Nitration of Easily Available Arylethanes}

\author{
$\mathrm{Mu}$, Bing $^{a, b} \quad$ Wu, Junliang ${ }^{*, a} \quad$ Zhang, Guang'an ${ }^{a}$ \\ ( ${ }^{a}$ College of Chemistry, Zhengzhou University, Zhengzhou 450001) \\ ${ }^{b}$ College of Chemistry and Chemical Engineering, Zhengzhou Normal University, Zhengzhou 450044)
}

\begin{abstract}
Nitroolefin is a common and versatile reagent, synthesis of which from aldehydes/ketones, $\alpha, \beta$-unsaturated carboxylic acids or olefins is generally limited by the high cost of raw materials in industrial processes in the future. Herein, an alternative and economical protocol for the synthesis of nitroaromatic olefins directly from easily available arylethanes with barium nitrate using $\mathrm{Cu} / \mathrm{Ag}$ as cocatalyst and ammonium persulfate as the terminal oxidant is reported. Additionally, 1,1-diphenylethanes, phenylethanes, 4-ethyl-1,1'-biphenyl and ethylnaphthalenes were suitable substrates for the current dehydrogenative nitration, and provided $E$-nitroaromatic olefins in moderate to good yields.

Keywords nitroaromatic olefins; arylethanes; barium nitrate; dehydrogenative nitration; ammonium persulfate
\end{abstract}

Nitroolefins are a versatile class of synthetic intermediates that have potential application in the preparation of a wide variety of pharmaceutical, biological, materials, and agrochemical relevant molecules. ${ }^{[1-5]}$ Besides, nitroalkenes are essential starting materials for various carbon-carbon bond forming reactions like Michael reaction, ${ }^{[6-10]} \mathrm{cy}-$ cloaddition, ${ }^{[11-17]}$ Morita-Baylis-Hillman reaction, ${ }^{[18-19]}$ and the generation of diverse chemically interesting molecules, such as oximes, nitroalkanes, hydroxylamines, aliphatic amines, and nitroso compounds. ${ }^{[20-21]}$

Consequently, considerable efforts have been made in the synthesis of nitroolefins, as shown in Scheme 1. These are conventionally synthesized by Henry reaction ${ }^{[22-23]}$ which relies upon base-mediated condensation of a nitroalkane with an aldehyde or ketone followed by subse- quent dehydration. However, harsh reaction conditions and formation of undesired $E / Z$ mixtures demand an alternative protocol. Recently, nitroalkenes are synthesized by the direct nitration of olefinic $\mathrm{C}-\mathrm{H}$ bonds with the nitrites, ${ }^{[24-32]}$ nitrogen dioxide, ${ }^{[33]}$ nitric oxide ${ }^{[34-36]}$ or claysupported nitrating reagents ${ }^{[37]}$ as well as by the decarboxylative nitration of $\alpha, \beta$-unsaturated carboxylic acids. ${ }^{[38-43]}$ The direct nitration of alkenes is considered as the preferred approach. In this direction, several synthetic methods for the nitration of olefins have been reported employing in situ generated $\mathrm{NO}_{2}$ centred radical from nitrites or nitrates in the presence of 2,2,6,6-tetramethylpiperidinooxy $(\mathrm{TEMPO})^{[44-47]}$ to achieve a better yield, give stereo-selective corresponding $E$-nitroolefins, avoid harsh conditions, and other difficulties associated with handling gaseous

* Corresponding author. E-mail: wuj1@zzu.edu.cn

Received August 24, 2020; revised November 27, 2020 ; published online January 7, 2021

Project supported by the National Natural Science Foundation of China (No. 21702191).

国家自然科学基金(No. 21702191)资助项目. 
reagents. Despite significant developments over the decade, there are limitations in direct nitration of olefins due to the cost and toxic nature of some of the reagent components as well as stoichiometric amounts of TEMPO.

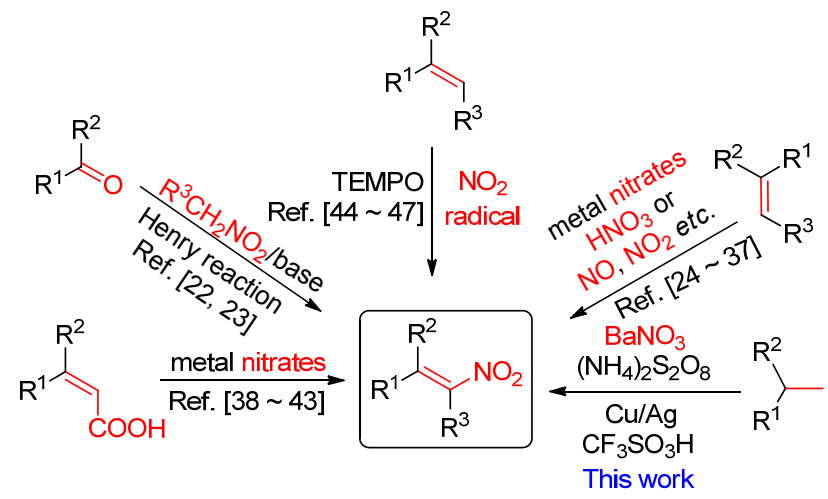

Scheme 1 Different approaches to synthesize nitroolefins

Considering the application of nitroolefins, development of an efficient, economical and practical method for the construction of nitroalkenes is highly desirable. Alkanes are principal constituents of fossil fuels such as coal, petroleum and natural gas, ${ }^{[48]}$ and are primary products of petroleum refining. ${ }^{[49]}$ Therefore, efficient conversion of such bulk chemicals into value-added synthetic intermediates is a critical task for future sustainable chemistry. ${ }^{[50-52]}$ It would be highly valuable if alkanes were used as starting materials to synthesize nitroalkenes by the chemoselective oxidation of $\mathrm{sp}^{3}-\mathrm{C}-\mathrm{H}$ bonds with the nitrates. Over the past decade, the oxygenation of $\mathrm{sp}^{3}-\mathrm{C}-\mathrm{H}$ bonds in a variety of substrates with $\mathrm{S}_{2} \mathrm{O}_{8}^{2-}$ and transition metals to generate an intermediate carbocation has been developed. ${ }^{[53-55]}$ The mechanistic studies have demonstrated that $\mathrm{sp}^{3}-\mathrm{C}-\mathrm{H}$ abstraction by $\mathrm{SO}_{4}^{--}$from $\mathrm{S}_{2} \mathrm{O}_{8}^{2-}$ affords an alkyl radical, then one-electron oxidation of the corresponding radical by transition metals leads to the formation of a resonance-stabilized carbocation, and in the presence of $\mathrm{S}_{2} \mathrm{O}_{8}^{2-}$, transition metal is re-oxidized, allowing for catalytic turnover. Intrigued by these findings, we speculated that if benzylic carbocation could be efficient generated from benzylic $\mathrm{C}-\mathrm{H}$ bonds, quick elimination might occur to give arylethylene, then the direct nitration of alkenes could be performed with nitrates. Antonchick and Burkhard's groups ${ }^{[56-57]}$ have reported the synthesis of nitrostyrenes by the dehydrogenative nitration of enyl toluenes, which only three examples have been performed. Herein, we attempt to develop a dehydrogenative nitration strategy to construct nitroaromatic olefins directly from easily available arylethanes with barium nitrate by using copper/silver as cocatalyst, and ammonium persulfate as the terminal oxidant.

\section{Results and discussion}

Initially, 1-ethylnaphthalene (1a) was selected as model substrate, and nitrites or nitrates as the nitro source to optimize the reaction conditions. Screening of the reaction conditions is shown in Table 1. At the outset of our studies, the effect of different reaction parameters on dehydrogenative nitration of 1-ethylnaphthalene was explored roughly, which included catalysts, ligands, oxidants, additives and solvents. Based on the rough experimental results, the model reaction was performed by reacting 1 a $(0.3 \mathrm{mmol})$ with 2.0 equiv. of $\mathrm{NaNO}_{2}$ in the presence of 0.05 equiv. of $\mathrm{CuBr}, 0.05$ equiv. of 4,7-diphenyl-1,10-phenathroline $\left(\mathbf{L}_{\mathbf{1}}\right)$, 1.5 equiv. of $\left(\mathrm{NH}_{4}\right)_{2} \mathrm{~S}_{2} \mathrm{O}_{8}, 0.2$ equiv. of $\mathrm{CF}_{3} \mathrm{COOAg}, 20$ equiv. of $\mathrm{CF}_{3} \mathrm{SO}_{3} \mathrm{H}$ and 1.0 equiv. of $\mathrm{K}_{3} \mathrm{PO}_{4}$ in $N, N^{\prime}$-dimethylformamide (DMF) at $100{ }^{\circ} \mathrm{C}$ for $16 \mathrm{~h}$. The formation of required product 1-[(E)-2-nitrovinyl]naphthalene (2a) was observed with the liquid chromatography (LC) yield of $30 \%$ (Entry 1). Further, a variety of the nitrating agents such as $\mathrm{NaNO}_{3}, \mathrm{KNO}_{3}, \mathrm{AgNO}_{2},{ }^{t} \mathrm{BuNO}_{2}$, $\mathrm{Bi}\left(\mathrm{NO}_{3}\right)_{3} \bullet 5 \mathrm{H}_{2} \mathrm{O}, \quad \mathrm{Ca}\left(\mathrm{NO}_{3}\right)_{2} \bullet 4 \mathrm{H}_{2} \mathrm{O}, \quad \mathrm{Cd}\left(\mathrm{NO}_{3}\right)_{2} \bullet 4 \mathrm{H}_{2} \mathrm{O}$, $\mathrm{Co}\left(\mathrm{NO}_{3}\right)_{2} \cdot 6 \mathrm{H}_{2} \mathrm{O}, \quad \mathrm{Ba}\left(\mathrm{NO}_{3}\right)_{2}$ and $\mathrm{Pb}\left(\mathrm{NO}_{3}\right)_{2}$ were also screened (Entries $2 \sim 11$ ). Obviously, $\mathrm{Ba}\left(\mathrm{NO}_{3}\right)_{2}$ and $\mathrm{Pb}\left(\mathrm{NO}_{3}\right)_{2}$ were identified as the better nitro sources in this reaction according to $80 \%$ and $86 \%$ LC yields, respectively (Entries 10 and 11 ). However, $\mathrm{Ba}\left(\mathrm{NO}_{3}\right)_{2}$ was chosen as nitro source for further studies because of avoiding heavy metal pollution. Whereas only poor yields of 2a were achieved using $\mathrm{CF}_{3} \mathrm{COOH}$, dioxane, 1,2-dichloroethane (DCE), dimethyl sulfoxide (DMSO) or $N, N$-dimethylacetamide (DMA) as solvent (Entries $12 \sim 16)$, no reaction occurred in $\mathrm{N}$-methyl pyrrolidone (NMP) (Entry 17). Then, the replacement of $\left(\mathrm{NH}_{4}\right)_{2} \mathrm{~S}_{2} \mathrm{O}_{8}$ with $\mathrm{Na}_{2} \mathrm{~S}_{2} \mathrm{O}_{8}, 2,3$-dichloro-5,6-dicyano-1,4-benzoquinone (DDQ), ${ }^{t} \mathrm{BuOO}{ }^{t} \mathrm{Bu}$ or ${ }^{t} \mathrm{BuOOH}$ leads to a drop in yield (Entries 18 21). Next, changing silver salts to $\mathrm{AgNO}_{3}, \mathrm{CH}_{3} \mathrm{COOAg}$ or $\mathrm{Ag}_{2} \mathrm{SO}_{4}$ afforded desired product in slightly decreased yields (Entries 22 24). However, no desired product was obtained in the case of $\mathrm{Ag}_{2} \mathrm{CO}_{3}$ (Entry 25). Moreover, the effect of other acids such as $\mathrm{CF}_{3} \mathrm{COOH}$, TsOH, ${ }^{t} \mathrm{BuCOOH}$ was also screened, and unfortunately, lower yields than that of $\mathrm{CF}_{3} \mathrm{SO}_{3} \mathrm{H}$ were observed (Entries 26 28). In addition, an examination of other ligands demonstrated that 2,2'-bipyridine $\left(\mathbf{L}_{2}\right)$, 1,10-phenanthroline $\left(\mathbf{L}_{3}\right)$, 4,7-dimethoxy1,10-phenanthroline $\left(\mathbf{L}_{\mathbf{4}}\right)$, 2,9-dimethyl-1,10-phenanthroline $\left(\mathbf{L}_{\mathbf{5}}\right)$ and 3,4,7,8-tetramethyl-1,10-phenanthroline $\left(\mathbf{L}_{\mathbf{6}}\right)$ are all usable ligands, providing $\mathbf{2 a}$ in slightly decreased yields (Entries 29 33). Finally, other catalyst such as $\mathrm{CuOAc}, \mathrm{CuI}, \mathrm{Cu}\left(\mathrm{CF}_{3} \mathrm{SO}_{3}\right)_{2},\left[\mathrm{CF}_{3} \mathrm{SO}_{3} \mathrm{Cu}\right]_{2} \cdot \mathrm{C}_{6} \mathrm{H}_{5} \mathrm{CH}_{3}, \mathrm{Cu}_{2} \mathrm{O}$, $\mathrm{CuCl}$ and $\mathrm{CuBr}_{2}$ were also employed, and $\mathrm{CuOAc}$ was identified as the best catalyst in this reaction according to 92\% LC yield (Entries 34 40). The desired product 2a was isolated in $89 \%$ isolated yield under the following optimized reaction conditions: 1-ethylnaphthalene $(0.3$ $\mathrm{mmol}), \mathrm{Ba}\left(\mathrm{NO}_{3}\right)_{2}(0.6 \mathrm{mmol}), \mathrm{CuOAc}(0.015 \mathrm{mmol}), 4,7-$ diphenyl-1,10-phenathroline $(0.015 \mathrm{mmol}),\left(\mathrm{NH}_{4}\right)_{2} \mathrm{~S}_{2} \mathrm{O}_{8}$ (0.45 mmol), $\mathrm{CF}_{3} \mathrm{COOAg}(0.06 \mathrm{mmol}), \mathrm{CF}_{3} \mathrm{SO}_{3} \mathrm{H}$ (6 $\mathrm{mmol}), \mathrm{K}_{3} \mathrm{PO}_{4}(0.3 \mathrm{mmol}), 2 \mathrm{~mL}$ of DMF, $100{ }^{\circ} \mathrm{C}, 16 \mathrm{~h}$, sealed tube.

The scope and generality of substrates for the dehydrogenative nitration of arylethane were investigated under the optimized conditions, and the results are summarized in 
Table 1 Optimization of the reaction conditions. ${ }^{a}$

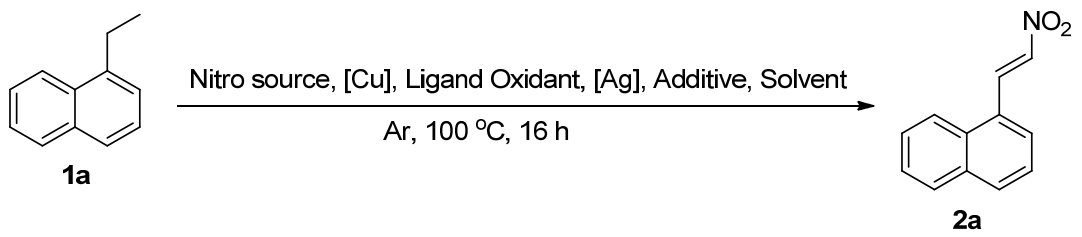

\begin{tabular}{|c|c|c|c|c|c|c|c|c|}
\hline Entry & Nitro source & {$[\mathrm{Cu}]$} & Ligand & Oxidant & {$[\mathrm{Ag}]$} & Additive & Solvent & Yield/\% \\
\hline 1 & $\mathrm{NaNO}_{2}$ & $\mathrm{CuBr}$ & $\mathbf{L}_{1}$ & $\left(\mathrm{NH}_{4}\right)_{2} \mathrm{~S}_{2} \mathrm{O}_{8}$ & $\mathrm{CF}_{3} \mathrm{COOAg}$ & $\mathrm{CF}_{3} \mathrm{SO}_{3} \mathrm{H}$ & DMF & 30 \\
\hline 2 & $\mathrm{NaNO}_{3}$ & $\mathrm{CuBr}$ & $\mathbf{L}_{1}$ & $\left(\mathrm{NH}_{4}\right)_{2} \mathrm{~S}_{2} \mathrm{O}_{8}$ & $\mathrm{CF}_{3} \mathrm{COOAg}$ & $\mathrm{CF}_{3} \mathrm{SO}_{3} \mathrm{H}$ & DMF & 66 \\
\hline 3 & $\mathrm{KNO}_{3}$ & $\mathrm{CuBr}$ & $\mathbf{L}_{1}$ & $\left(\mathrm{NH}_{4}\right)_{2} \mathrm{~S}_{2} \mathrm{O}_{8}$ & $\mathrm{CF}_{3} \mathrm{COOAg}$ & $\mathrm{CF}_{3} \mathrm{SO}_{3} \mathrm{H}$ & DMF & 56 \\
\hline 4 & $\mathrm{AgNO}_{2}$ & $\mathrm{CuBr}$ & $\mathbf{L}_{1}$ & $\left(\mathrm{NH}_{4}\right)_{2} \mathrm{~S}_{2} \mathrm{O}_{8}$ & $\mathrm{CF}_{3} \mathrm{COOAg}$ & $\mathrm{CF}_{3} \mathrm{SO}_{3} \mathrm{H}$ & DMF & 39 \\
\hline 5 & ${ }^{t} \mathrm{BuNO}_{2}$ & $\mathrm{CuBr}$ & $\mathbf{L}_{1}$ & $\left(\mathrm{NH}_{4}\right)_{2} \mathrm{~S}_{2} \mathrm{O}_{8}$ & $\mathrm{CF}_{3} \mathrm{COOAg}$ & $\mathrm{CF}_{3} \mathrm{SO}_{3} \mathrm{H}$ & DMF & 8 \\
\hline 6 & $\mathrm{Bi}\left(\mathrm{NO}_{3}\right)_{3} \cdot 5 \mathrm{H}_{2} \mathrm{O}$ & $\mathrm{CuBr}$ & $\mathbf{L}_{1}$ & $\left(\mathrm{NH}_{4}\right)_{2} \mathrm{~S}_{2} \mathrm{O}_{8}$ & $\mathrm{CF}_{3} \mathrm{COOAg}$ & $\mathrm{CF}_{3} \mathrm{SO}_{3} \mathrm{H}$ & DMF & 57 \\
\hline 7 & $\mathrm{Ca}\left(\mathrm{NO}_{3}\right)_{2} \bullet 4 \mathrm{H}_{2} \mathrm{O}$ & $\mathrm{CuBr}$ & $\mathbf{L}_{1}$ & $\left(\mathrm{NH}_{4}\right)_{2} \mathrm{~S}_{2} \mathrm{O}_{8}$ & $\mathrm{CF}_{3} \mathrm{COOAg}$ & $\mathrm{CF}_{3} \mathrm{SO}_{3} \mathrm{H}$ & DMF & 51 \\
\hline 8 & $\mathrm{Cd}\left(\mathrm{NO}_{3}\right)_{2} \bullet 4 \mathrm{H}_{2} \mathrm{O}$ & $\mathrm{CuBr}$ & $\mathbf{L}_{1}$ & $\left(\mathrm{NH}_{4}\right)_{2} \mathrm{~S}_{2} \mathrm{O}_{8}$ & $\mathrm{CF}_{3} \mathrm{COOAg}$ & $\mathrm{CF}_{3} \mathrm{SO}_{3} \mathrm{H}$ & DMF & 54 \\
\hline 9 & $\mathrm{Co}\left(\mathrm{NO}_{3}\right)_{2} \cdot 6 \mathrm{H}_{2} \mathrm{O}$ & $\mathrm{CuBr}$ & $\mathbf{L}_{1}$ & $\left(\mathrm{NH}_{4}\right)_{2} \mathrm{~S}_{2} \mathrm{O}_{8}$ & $\mathrm{CF}_{3} \mathrm{COOAg}$ & $\mathrm{CF}_{3} \mathrm{SO}_{3} \mathrm{H}$ & DMF & 25 \\
\hline 10 & $\mathrm{Ba}\left(\mathrm{NO}_{3}\right)_{2}$ & $\mathrm{CuBr}$ & $\mathbf{L}_{1}$ & $\left(\mathrm{NH}_{4}\right)_{2} \mathrm{~S}_{2} \mathrm{O}_{8}$ & $\mathrm{CF}_{3} \mathrm{COOAg}$ & $\mathrm{CF}_{3} \mathrm{SO}_{3} \mathrm{H}$ & DMF & 80 \\
\hline 11 & $\mathrm{~Pb}\left(\mathrm{NO}_{3}\right)_{2}$ & $\mathrm{CuBr}$ & $\mathbf{L}_{1}$ & $\left(\mathrm{NH}_{4}\right)_{2} \mathrm{~S}_{2} \mathrm{O}_{8}$ & $\mathrm{CF}_{3} \mathrm{COOAg}$ & $\mathrm{CF}_{3} \mathrm{SO}_{3} \mathrm{H}$ & DMF & 86 \\
\hline 12 & $\mathrm{Ba}\left(\mathrm{NO}_{3}\right)_{2}$ & $\mathrm{CuBr}$ & $\mathbf{L}_{1}$ & $\left(\mathrm{NH}_{4}\right)_{2} \mathrm{~S}_{2} \mathrm{O}_{8}$ & $\mathrm{CF}_{3} \mathrm{COOAg}$ & $\mathrm{CF}_{3} \mathrm{SO}_{3} \mathrm{H}$ & $\mathrm{CF}_{3} \mathrm{COOH}$ & 22 \\
\hline 13 & $\mathrm{Ba}\left(\mathrm{NO}_{3}\right)_{2}$ & $\mathrm{CuBr}$ & $\mathbf{L}_{1}$ & $\left(\mathrm{NH}_{4}\right)_{2} \mathrm{~S}_{2} \mathrm{O}_{8}$ & $\mathrm{CF}_{3} \mathrm{COOAg}$ & $\mathrm{CF}_{3} \mathrm{SO}_{3} \mathrm{H}$ & Dioxane & 11 \\
\hline 14 & $\mathrm{Ba}\left(\mathrm{NO}_{3}\right)_{2}$ & $\mathrm{CuBr}$ & $\mathbf{L}_{1}$ & $\left(\mathrm{NH}_{4}\right)_{2} \mathrm{~S}_{2} \mathrm{O}_{8}$ & $\mathrm{CF}_{3} \mathrm{COOAg}$ & $\mathrm{CF}_{3} \mathrm{SO}_{3} \mathrm{H}$ & DCE & 12 \\
\hline 15 & $\mathrm{Ba}\left(\mathrm{NO}_{3}\right)_{2}$ & $\mathrm{CuBr}$ & $\mathbf{L}_{1}$ & $\left(\mathrm{NH}_{4}\right)_{2} \mathrm{~S}_{2} \mathrm{O}_{8}$ & $\mathrm{CF}_{3} \mathrm{COOAg}$ & $\mathrm{CF}_{3} \mathrm{SO}_{3} \mathrm{H}$ & DMSO & 14 \\
\hline 16 & $\mathrm{Ba}\left(\mathrm{NO}_{3}\right)_{2}$ & $\mathrm{CuBr}$ & $\mathbf{L}_{1}$ & $\left(\mathrm{NH}_{4}\right)_{2} \mathrm{~S}_{2} \mathrm{O}_{8}$ & $\mathrm{CF}_{3} \mathrm{COOAg}$ & $\mathrm{CF}_{3} \mathrm{SO}_{3} \mathrm{H}$ & DMA & 8 \\
\hline 17 & $\mathrm{Ba}\left(\mathrm{NO}_{3}\right)_{2}$ & $\mathrm{CuBr}$ & $\mathbf{L}_{1}$ & $\left(\mathrm{NH}_{4}\right)_{2} \mathrm{~S}_{2} \mathrm{O}_{8}$ & $\mathrm{CF}_{3} \mathrm{COOAg}$ & $\mathrm{CF}_{3} \mathrm{SO}_{3} \mathrm{H}$ & NMP & NR \\
\hline 18 & $\mathrm{Ba}\left(\mathrm{NO}_{3}\right)_{2}$ & $\mathrm{CuBr}$ & $\mathbf{L}_{1}$ & $\mathrm{Na}_{2} \mathrm{~S}_{2} \mathrm{O}_{8}$ & $\mathrm{CF}_{3} \mathrm{COOAg}$ & $\mathrm{CF}_{3} \mathrm{SO}_{3} \mathrm{H}$ & DMF & 59 \\
\hline 19 & $\mathrm{Ba}\left(\mathrm{NO}_{3}\right)_{2}$ & $\mathrm{CuBr}$ & $\mathbf{L}_{1}$ & DDQ & $\mathrm{CF}_{3} \mathrm{COOAg}$ & $\mathrm{CF}_{3} \mathrm{SO}_{3} \mathrm{H}$ & DMF & 10 \\
\hline 20 & $\mathrm{Ba}\left(\mathrm{NO}_{3}\right)_{2}$ & $\mathrm{CuBr}$ & $\mathbf{L}_{1}$ & ${ }^{t} \mathrm{BuOO}{ }^{t} \mathrm{Bu}$ & $\mathrm{CF}_{3} \mathrm{COOAg}$ & $\mathrm{CF}_{3} \mathrm{SO}_{3} \mathrm{H}$ & DMF & 6 \\
\hline 21 & $\mathrm{Ba}\left(\mathrm{NO}_{3}\right)_{2}$ & $\mathrm{CuBr}$ & $\mathbf{L}_{1}$ & ${ }^{t} \mathrm{BuOOH}$ & $\mathrm{CF}_{3} \mathrm{COOAg}$ & $\mathrm{CF}_{3} \mathrm{SO}_{3} \mathrm{H}$ & DMF & NR \\
\hline 22 & $\mathrm{Ba}\left(\mathrm{NO}_{3}\right)_{2}$ & $\mathrm{CuBr}$ & $\mathbf{L}_{1}$ & $\left(\mathrm{NH}_{4}\right)_{2} \mathrm{~S}_{2} \mathrm{O}_{8}$ & $\mathrm{AgNO}_{3}$ & $\mathrm{CF}_{3} \mathrm{SO}_{3} \mathrm{H}$ & DMF & 78 \\
\hline 23 & $\mathrm{Ba}\left(\mathrm{NO}_{3}\right)_{2}$ & $\mathrm{CuBr}$ & $\mathbf{L}_{1}$ & $\left(\mathrm{NH}_{4}\right)_{2} \mathrm{~S}_{2} \mathrm{O}_{8}$ & $\mathrm{CH}_{3} \mathrm{COOAg}$ & $\mathrm{CF}_{3} \mathrm{SO}_{3} \mathrm{H}$ & DMF & 75 \\
\hline 24 & $\mathrm{Ba}\left(\mathrm{NO}_{3}\right)_{2}$ & $\mathrm{CuBr}$ & $\mathbf{L}_{1}$ & $\left(\mathrm{NH}_{4}\right)_{2} \mathrm{~S}_{2} \mathrm{O}_{8}$ & $\mathrm{Ag}_{2} \mathrm{SO}_{4}$ & $\mathrm{CF}_{3} \mathrm{SO}_{3} \mathrm{H}$ & DMF & 73 \\
\hline 25 & $\mathrm{Ba}\left(\mathrm{NO}_{3}\right)_{2}$ & $\mathrm{CuBr}$ & $\mathbf{L}_{1}$ & $\left(\mathrm{NH}_{4}\right)_{2} \mathrm{~S}_{2} \mathrm{O}_{8}$ & $\mathrm{Ag}_{2} \mathrm{CO}_{3}$ & $\mathrm{CF}_{3} \mathrm{SO}_{3} \mathrm{H}$ & DMF & NR \\
\hline 26 & $\mathrm{Ba}\left(\mathrm{NO}_{3}\right)_{2}$ & $\mathrm{CuBr}$ & $\mathbf{L}_{1}$ & $\left(\mathrm{NH}_{4}\right)_{2} \mathrm{~S}_{2} \mathrm{O}_{8}$ & $\mathrm{CF}_{3} \mathrm{COOAg}$ & $\mathrm{CF}_{3} \mathrm{COOH}$ & DMF & 42 \\
\hline 27 & $\mathrm{Ba}\left(\mathrm{NO}_{3}\right)_{2}$ & $\mathrm{CuBr}$ & $\mathbf{L}_{1}$ & $\left(\mathrm{NH}_{4}\right)_{2} \mathrm{~S}_{2} \mathrm{O}_{8}$ & $\mathrm{CF}_{3} \mathrm{COOAg}$ & $\mathrm{TsOH}$ & DMF & 16 \\
\hline 28 & $\mathrm{Ba}\left(\mathrm{NO}_{3}\right)_{2}$ & $\mathrm{CuBr}$ & $\mathbf{L}_{1}$ & $\left(\mathrm{NH}_{4}\right)_{2} \mathrm{~S}_{2} \mathrm{O}_{8}$ & $\mathrm{CF}_{3} \mathrm{COOAg}$ & ${ }^{t} \mathrm{BuCOOH}$ & DMF & 27 \\
\hline 29 & $\mathrm{Ba}\left(\mathrm{NO}_{3}\right)_{2}$ & $\mathrm{CuBr}$ & $\mathbf{L}_{2}$ & $\left(\mathrm{NH}_{4}\right)_{2} \mathrm{~S}_{2} \mathrm{O}_{8}$ & $\mathrm{CF}_{3} \mathrm{COOAg}$ & $\mathrm{CF}_{3} \mathrm{SO}_{3} \mathrm{H}$ & DMF & 75 \\
\hline 30 & $\mathrm{Ba}\left(\mathrm{NO}_{3}\right)_{2}$ & $\mathrm{CuBr}$ & $\mathbf{L}_{3}$ & $\left(\mathrm{NH}_{4}\right)_{2} \mathrm{~S}_{2} \mathrm{O}_{8}$ & $\mathrm{CF}_{3} \mathrm{COOAg}$ & $\mathrm{CF}_{3} \mathrm{SO}_{3} \mathrm{H}$ & DMF & 76 \\
\hline 31 & $\mathrm{Ba}\left(\mathrm{NO}_{3}\right)_{2}$ & $\mathrm{CuBr}$ & $\mathbf{L}_{4}$ & $\left(\mathrm{NH}_{4}\right)_{2} \mathrm{~S}_{2} \mathrm{O}_{8}$ & $\mathrm{CF}_{3} \mathrm{COOAg}$ & $\mathrm{CF}_{3} \mathrm{SO}_{3} \mathrm{H}$ & DMF & 64 \\
\hline 32 & $\mathrm{Ba}\left(\mathrm{NO}_{3}\right)_{2}$ & $\mathrm{CuBr}$ & $\mathbf{L}_{5}$ & $\left(\mathrm{NH}_{4}\right)_{2} \mathrm{~S}_{2} \mathrm{O}_{8}$ & $\mathrm{CF}_{3} \mathrm{COOAg}$ & $\mathrm{CF}_{3} \mathrm{SO}_{3} \mathrm{H}$ & DMF & 72 \\
\hline 33 & $\mathrm{Ba}\left(\mathrm{NO}_{3}\right)_{2}$ & $\mathrm{CuBr}$ & $\mathbf{L}_{6}$ & $\left(\mathrm{NH}_{4}\right)_{2} \mathrm{~S}_{2} \mathrm{O}_{8}$ & $\mathrm{CF}_{3} \mathrm{COOAg}$ & $\mathrm{CF}_{3} \mathrm{SO}_{3} \mathrm{H}$ & DMF & 79 \\
\hline 34 & $\mathrm{Ba}\left(\mathrm{NO}_{3}\right)_{2}$ & $\mathrm{CuOAc}$ & $\mathbf{L}_{1}$ & $\left(\mathrm{NH}_{4}\right)_{2} \mathrm{~S}_{2} \mathrm{O}_{8}$ & $\mathrm{CF}_{3} \mathrm{COOAg}$ & $\mathrm{CF}_{3} \mathrm{SO}_{3} \mathrm{H}$ & DMF & $92(89)^{b}$ \\
\hline 35 & $\mathrm{Ba}\left(\mathrm{NO}_{3}\right)_{2}$ & $\mathrm{CuI}$ & $\mathbf{L}_{1}$ & $\left(\mathrm{NH}_{4}\right)_{2} \mathrm{~S}_{2} \mathrm{O}_{8}$ & $\mathrm{CF}_{3} \mathrm{COOAg}$ & $\mathrm{CF}_{3} \mathrm{SO}_{3} \mathrm{H}$ & DMF & 80 \\
\hline 36 & $\mathrm{Ba}\left(\mathrm{NO}_{3}\right)_{2}$ & $\left(\mathrm{CF}_{3} \mathrm{SO}_{3}\right)_{2} \mathrm{Cu}$ & $\mathbf{L}_{1}$ & $\left(\mathrm{NH}_{4}\right)_{2} \mathrm{~S}_{2} \mathrm{O}_{8}$ & $\mathrm{CF}_{3} \mathrm{COOAg}$ & $\mathrm{CF}_{3} \mathrm{SO}_{3} \mathrm{H}$ & DMF & 80 \\
\hline 37 & $\mathrm{Ba}\left(\mathrm{NO}_{3}\right)_{2}$ & $\begin{array}{l}{\left[\mathrm{CF}_{3} \mathrm{SO}_{3} \mathrm{Cu}\right]_{2} \bullet} \\
\mathrm{C}_{6} \mathrm{H}_{5} \mathrm{CH}_{3}\end{array}$ & $\mathbf{L}_{1}$ & $\left(\mathrm{NH}_{4}\right)_{2} \mathrm{~S}_{2} \mathrm{O}_{8}$ & $\mathrm{CF}_{3} \mathrm{COOAg}$ & $\mathrm{CF}_{3} \mathrm{SO}_{3} \mathrm{H}$ & DMF & 82 \\
\hline 38 & $\mathrm{Ba}\left(\mathrm{NO}_{3}\right)_{2}$ & $\mathrm{Cu}_{2} \mathrm{O}$ & $\mathbf{L}_{1}$ & $\left(\mathrm{NH}_{4}\right)_{2} \mathrm{~S}_{2} \mathrm{O}_{8}$ & $\mathrm{CF}_{3} \mathrm{COOAg}$ & $\mathrm{CF}_{3} \mathrm{SO}_{3} \mathrm{H}$ & DMF & 73 \\
\hline 39 & $\mathrm{Ba}\left(\mathrm{NO}_{3}\right)_{2}$ & $\mathrm{CuCl}$ & $\mathbf{L}_{1}$ & $\left(\mathrm{NH}_{4}\right)_{2} \mathrm{~S}_{2} \mathrm{O}_{8}$ & $\mathrm{CF}_{3} \mathrm{COOAg}$ & $\mathrm{CF}_{3} \mathrm{SO}_{3} \mathrm{H}$ & DMF & 76 \\
\hline 40 & $\mathrm{Ba}\left(\mathrm{NO}_{3}\right)_{2}$ & $\mathrm{CuBr}_{2}$ & $\mathbf{L}_{1}$ & $\left(\mathrm{NH}_{4}\right)_{2} \mathrm{~S}_{2} \mathrm{O}_{8}$ & $\mathrm{CF}_{3} \mathrm{COOAg}$ & $\mathrm{CF}_{3} \mathrm{SO}_{3} \mathrm{H}$ & DMF & 77 \\
\hline
\end{tabular}

${ }^{a}$ Reaction conditions: 1a $(0.3 \mathrm{mmol}),[\mathrm{Cu}]\left(0.05\right.$ equiv.), ligand ( 0.05 equiv.), oxidant ( 1.5 equiv.), $[\mathrm{Ag}]^{+}$(0.2 equiv.), additive (20 equiv.), nitro source ( 2 equiv.), $\mathrm{K}_{3} \mathrm{PO}_{4}$ (1 equiv.), DMF (2 mL), Argon, $100{ }^{\circ} \mathrm{C}$, LC yield, $16 \mathrm{~h} .{ }^{b}$ Isolated yield.

Table 2. Firstly, 2-ethylnaphthalene with barium nitrate reacted under the optimized reaction conditions, generating the corresponding product $\mathbf{2 b}$ in lower yield of $52 \%$. Then, the reactions between substituted naphthylethane and bar- ium nitrate were also conducted. 1-Ethyl-5-methoxynaphthalene bearing strong electron-donating group proved to be challenging substrate, and no desired product $\mathbf{2 c}$ was detected by GC-MS analysis. This is a result of competing 
reaction such as nitration of the aromatic ring, which gave large amounts of nitration products. When the sterically hindered 1-isopropylnaphthalene was checked, no desired product 2d was obtained. So it was deduced that the steric Table 2 Dehydrogenative nitration of arylethanes with barium nitrate

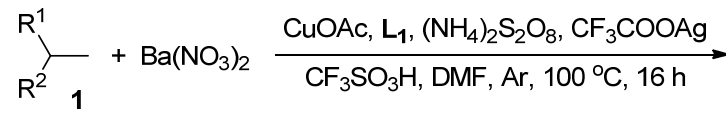

$$
\begin{aligned}
& \mathrm{R}^{1} \\
& \mathrm{R}_{2}^{2} \mathrm{NO}_{2} \quad \mathrm{R}^{1}=\mathrm{Ar} ; \mathrm{R}^{2}=\mathrm{Ar}, \mathrm{H}
\end{aligned}
$$

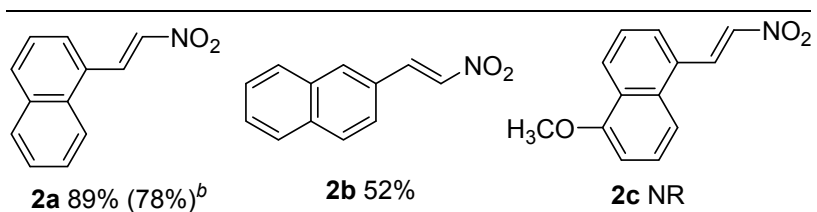<smiles>COc1ccc(C(=C[N+](=O)[O-])c2ccc(OC)cc2)cc1</smiles>

2d NR

2e $84 \%$<smiles>COc1ccccc1C(C[N+](=O)[O-])c1ccccc1</smiles><smiles>Cc1ccc(C=Cc2ccc(C)cc2)cc1</smiles>

2h $48 \%$<smiles>COc1ccc(C(=C[N+](=O)[O-])c2ccc(OC)cc2)cc1</smiles><smiles>COc1ccc(C(=C[N+](=O)[O-])c2ccc(Cl)cc2)cc1</smiles><smiles>O=[N+]([O-])C=C(c1ccccc1)c1ccc(Cl)cc1</smiles>

2k $31 \%$<smiles>COc1ccc(/C=C/[N+](=O)[O-])cc1</smiles>

2n $74 \%$

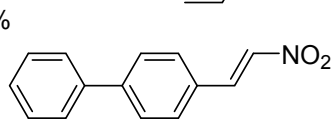

2p $37 \%(44 \%)^{d}$

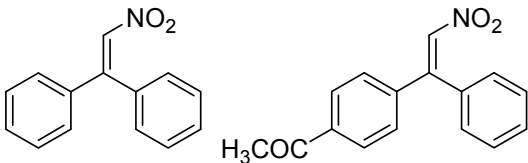

2I $55 \%^{\mathrm{c}}$

$2 \mathrm{~m}$ Trace

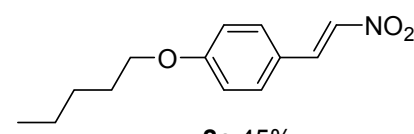

$2045 \%$
${ }^{a}$ Reaction conditions: arylethane $1(0.3 \mathrm{mmol}), \mathrm{Ba}\left(\mathrm{NO}_{3}\right)_{2}(0.6 \mathrm{mmol}), \mathrm{CuOAc}$ (0.015 mmol), $\mathbf{L}_{\mathbf{1}}(0.015 \mathrm{mmol}),\left(\mathrm{NH}_{4}\right)_{2} \mathrm{~S}_{2} \mathrm{O}_{8}(0.45 \mathrm{mmol}), \mathrm{CF}_{3} \mathrm{COOAg}(0.06$ $\mathrm{mmol}), \mathrm{CF}_{3} \mathrm{SO}_{3} \mathrm{H}(6 \mathrm{mmol}), \mathrm{K}_{3} \mathrm{PO}_{4}(0.3 \mathrm{mmol}), \mathrm{DMF}(2 \mathrm{~mL})$, Argon, $100{ }^{\circ} \mathrm{C}$, $16 \mathrm{~h}$, isolated yield. ${ }^{b} 6.6 \mathrm{mmol}(1.030 \mathrm{~g})$ of 1-ethylnaphthalene. ${ }^{c}\left(\mathrm{CH}_{3} \mathrm{CN}\right)_{4}$ $\mathrm{CuPF}_{6}(0.015 \mathrm{mmol}), \mathrm{CF}_{3} \mathrm{SO}_{3} \mathrm{H}(1 \mathrm{~mL}) .{ }^{d} 110{ }^{\circ} \mathrm{C}$.

hindrance of isopropyl substituent could have influence on the dehydrogenative nitration. Next, the applicability of dehydrogenative nitration protocol with 1,1-diphenylethanes was examined. As expected, the substrates containing electron-donating group could reacted under the optimized reaction conditions, generating the correspond- ing products $\mathbf{2 e} \sim \mathbf{2 g}$ in $61 \% \sim 84 \%$ yields. However, 1methoxy-2-[1-(4-methoxyphenyl)ethyl]benzene gave corresponding product $2 \mathbf{f}$ in yield of $81 \%$ with $E / Z$ ratio of $10: 1$. The reaction with 1-methoxy-2-(1-phenylethyl)benzene, 4,4'-(ethane-1,1-diyl)bis(methoxybenzene), 1-chloro4-[1-(4-methoxyphenyl)ethyl]benzene, 1-chloro-4-(1-phenylethyl)benzene gave the desired product $\mathbf{2 h} \sim \mathbf{2 k}$ in $31 \% \sim 48 \%$ yields. For 4,4'-(ethane-1,1-diyl)bis(methoxybenzene) containing strong electron-donating group, the desired product $2 \mathbf{i}$ was obtained in lower yield of $43 \%$ due to the nitration of aromatic ring. Moreover using 1-chloro4-(1-phenylethyl)benzene as substrate led to the formation of $E / Z$ mixture of the corresponding product $\mathbf{2 k}$. When TfOH loading was increased to $1 \mathrm{~mL}$ with $\left(\mathrm{CH}_{3} \mathrm{CN}\right)_{4^{-}}$ $\mathrm{CuPF}_{6}$ as catalyst, the dehydrogenative nitration of 1,1 diphenylethane afforded the desired product $2 \mathrm{l}$ in $55 \%$ yield. To our disappointment, when 1-[4-(1-phenyl-ethyl)phenyl]ethanone was introduced into the reaction system, only a trace amount of the product $\mathbf{2} \mathbf{m}$ was detected by GC-MS analysis probably because the electron-withdrawing effect was not beneficial to the stability of intermediates. In addition, it was pleasing to find that the reaction was successful with 1-ethyl-4-methoxybenzene or 1-ethyl4-(pentyloxy)benzene with barium nitrate, which gave the desired products $2 \mathrm{n}$ and $\mathbf{2 0}$ in $74 \%$ and $45 \%$ yields, respectively. Finally, when the dehydrogenative nitration of 4-ethylbiphenyl was performed at a higher temperature of $110{ }^{\circ} \mathrm{C}$, the yield increased to $44 \%$ yield (product $\mathbf{2 p}$ ). To demonstrate the scalability of the reaction, the dehydrogenative nitration of 1-ethylnaphthalene with $\mathrm{Ba}\left(\mathrm{NO}_{3}\right)_{2}$ on a $1.0 \mathrm{~g}$ scale was chosen. The desired product was isolated in $78 \%$ yield (product $\mathbf{2 a}$ ).

To gain insight into the mechanism, controlled experiments were carried out under different reaction conditions. The results are presented in Scheme 2. First of all, to explore the necessity of $\mathrm{CF}_{3} \mathrm{SO}_{3} \mathrm{H}$ as the reagent partner, the reactions were also carried with varying amounts of $\mathrm{CF}_{3} \mathrm{SO}_{3} \mathrm{H}$. Lower amounts of $\mathrm{CF}_{3} \mathrm{SO}_{3} \mathrm{H}$ resulted in significant decline in the yield of the desired product (Scheme 2a). Moreover, when the reaction was conducted in the absence of $\mathrm{CF}_{3} \mathrm{SO}_{3} \mathrm{H}$, the conversion sharply dropped to $9 \%$ (Scheme $2 \mathrm{~b}$ ), clearly demonstrating the crucial role of $\mathrm{CF}_{3} \mathrm{SO}_{3} \mathrm{H}$ in improving the efficiency of the reactions significantly. Encouraged by this result, $\mathrm{HNO}_{3}$ was tested as nitro source and proton source, and unfortunately, only poor yields of 2a were achieved (Schemes $2 \mathrm{c}$ and $2 \mathrm{~d}$ ). In addition, the product $\mathbf{2 a}$ was obtained in $50 \%$ LC yield without $\mathrm{CF}_{3} \mathrm{COOAg}$, and in an LC yield of $20 \%$ without $\mathrm{CuOAc}$ and 4,7-diphenyl-1,10-phenathroline (Schemes 2e and $2 \mathrm{f}$ ). The results indirectly indicated that the catalytic amount of $\mathrm{CuOAc}$ and $\mathrm{CF}_{3} \mathrm{COOAg}$ as cocatalyst, 4,7-diphenyl-1,10-phenathroline as ligand, and $\left(\mathrm{NH}_{4}\right)_{2} \mathrm{~S}_{2} \mathrm{O}_{8}$ as oxidant may play a crucial role in dehydrogenative nitration. Comparative experiments with or without $\mathrm{K}_{3} \mathrm{PO}_{4}$ confirmed its necessity to obtain the desired outcome of the dehydrogenative nitration (Scheme $2 \mathrm{~g}$ ). $\mathrm{K}_{3} \mathrm{PO}_{4}$ as an additive in the presence of $\mathrm{CF}_{3} \mathrm{SO}_{3} \mathrm{H}$ produced $\mathrm{H}_{2} \mathrm{PO}_{4}^{-}$ 
and $\mathrm{HPO}_{4}^{2-}$ ions, which was probably positive for generating free radicals. ${ }^{[58]}$ Finally, radical trapping experiments were conducted using 2,2,6,6-tetramethylpiperidine1-oxyl (TEMPO) or butylated hydroxytoluene (BHT) as the radical scavenger (Schemes $2 \mathrm{~h}$ and $2 \mathrm{i}$ ). In the presence of 1 equiv. of TEMPO or BHT, the reaction of 1-ethylnaphthalene (1a) and $\mathrm{Ba}\left(\mathrm{NO}_{3}\right)_{2}$ was effectively suppressed under standard conditions, suggesting that radical intermediates might be involved in this transformation. Finally, 1vinylnaphthalene was added instead of 1-ethylnaphthalene, and the reaction was allowed to occur under standard conditions (Scheme 2j). However, the desired product 2a was detected in only $5 \%$ yield, although the complete consumption of 1-vinylnaphthalene was observed, probably because of the oxidative decomposition of 1-vinylnaphthalene under the relatively harsh reaction conditions.

(a) 15 equiv. $\mathrm{CF}_{3} \mathrm{SO}_{3} \mathrm{H}$
(b) without $\mathrm{CF}_{3} \mathrm{SO}_{3} \mathrm{H}$
(c) 20 equiv. $\mathrm{HNO}_{3}$ without $\mathrm{CF}_{3} \mathrm{SO}_{3} \mathrm{H}$ and $\mathrm{Ba}\left(\mathrm{NO}_{3}\right)_{2}$

Scheme 2 Comparative experiments under different reaction conditions

On the basis of the above-mentioned experimental results and previous literature reports, ${ }^{[44,53,55-57,59]}$ a possible mechanism of $\mathrm{Cu} / \mathrm{Ag}$-cocatalyzed dehydrogenative nitration by using 1-ethylnaphthalene (1a) and $\mathrm{Ba}\left(\mathrm{NO}_{3}\right)_{2}$ as an example is outlined in Scheme 3. Initially, sulfate radical $\mathrm{SO}_{4}{ }^{-\cdot}$ can be generated from persulfate $\mathrm{S}_{2} \mathrm{O}_{8}^{2-}$, and is known to react rapidly with saturated hydrocarbons. ${ }^{[60-61]}$
Then, benzylic hydrogen abstraction in 1-ethylnaphthalene by $\mathrm{SO}_{4}^{-}$affords a carbon radical intermediate $\mathbf{I}$. The radical I could be oxidized into a carbocation II by the action of $\mathrm{Ag}(\mathrm{II}) / \mathrm{Cu}(\mathrm{II})$, which was generated from $\mathrm{Ag}(\mathrm{I}) /$ $\mathrm{Cu}(\mathrm{I})$ through oxidation by $\left(\mathrm{NH}_{4}\right)_{2} \mathrm{~S}_{2} \mathrm{O}_{8} \cdot{ }^{[62]}$ The formed carbocation II could be trapped by $\mathrm{CF}_{3} \mathrm{SO}_{3}^{-}$, then a $\mathrm{CF}_{3} \mathrm{SO}_{3} \mathrm{H}$ elimination step furnishes 1-vinylnaphthalene III. In the control experiment, the target product of dehydrogenative nitration was not obtained using 1-vinylnaphthalene instead of 1-ethylnaphthalene (Scheme 2j), probably because the oxidative decomposition occurred under the relatively harsh reaction conditions when a large amount of 1-vinylnaphthalene was added into the reaction system. However, the intermediate 1-vinylnaphthalene produced instantaneously in the reaction system is likely to be underwent rapidly the dehydrogenative nitration to give the target product under the optimized conditions. $\mathrm{CF}_{3} \mathrm{SO}_{3} \mathrm{H}$ might act as an additive to stabilize the carbocations and promote the deprotonation to generate alkenes. Subsequently, nitro radical may be generated from $\mathrm{Ba}\left(\mathrm{NO}_{3}\right)_{2}$ under the applied reaction condition. Thereafter, the nitro radical would react at the less-hindered side of the olefin in order to generate a benzylic radical IV in terms of stability of the radical, thereby determining regioselectivity of the reaction solely. In the last step, the radical IV could be oxidized into a carbocation $\mathbf{V}$ by the action of $\operatorname{Ag}(\mathrm{II}) /$ $\mathrm{Cu}(\mathrm{II})$, which went through deprotonation to produce product 2a. The utilization of $\mathrm{CF}_{3} \mathrm{SO}_{3} \mathrm{H}$ as an additive could be positive for the deprotonation to release the nitroolefin product. The evident role of $\mathrm{CF}_{3} \mathrm{SO}_{3} \mathrm{H}$ and $\mathrm{K}_{3} \mathrm{PO}_{4}$ on reaction efficiency is clear from the data shown in Scheme 2. Future studies will aim to elucidate their unique function in this process.

\section{Conclusions}

In summary, a novel and economical method for the synthesis of $E$-nitroaromatic olefins has been developed via the copper/silver cocatalyzed dehydrogenative nitration 


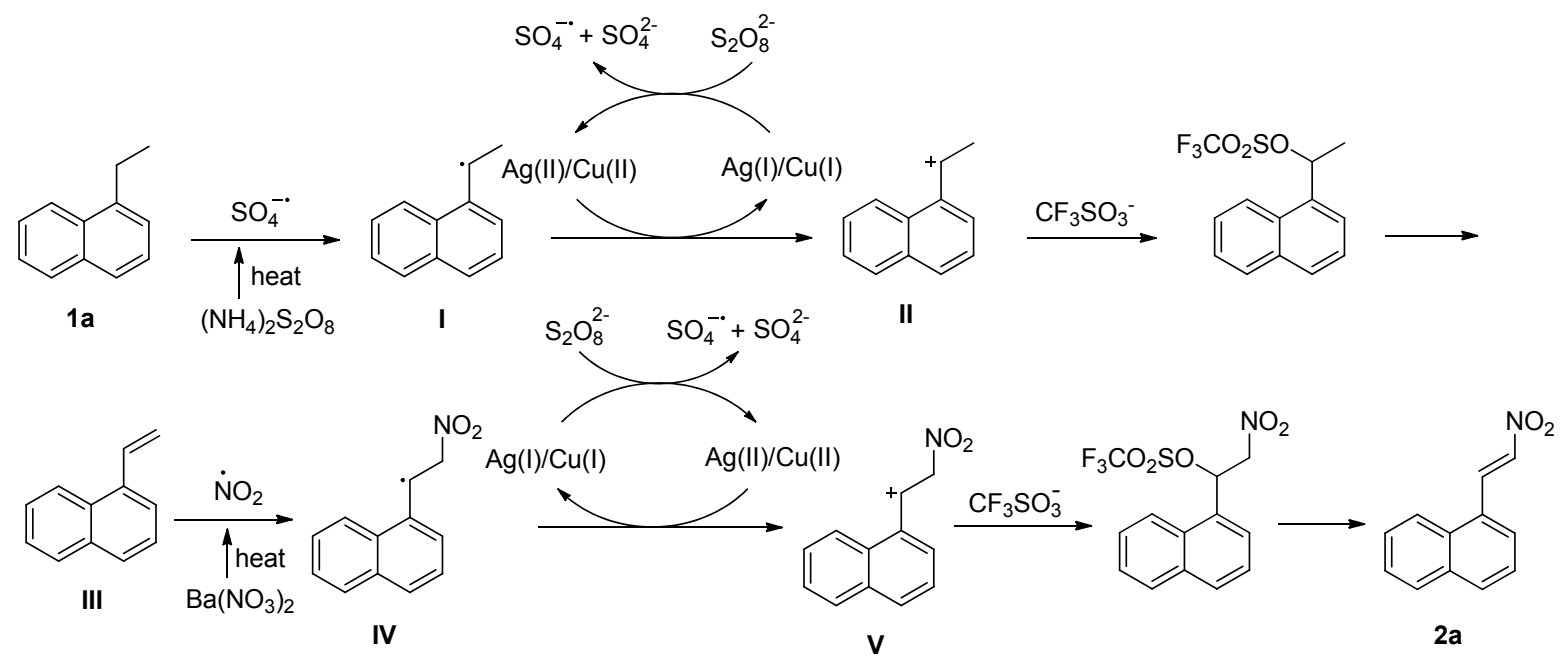

Scheme 3 Proposed mechanism of dehydrogenative nitration of 1-ethylnaphthalene with $\mathrm{Ba}\left(\mathrm{NO}_{3}\right)_{2}$

of easily available arylethanes with barium nitrate in the presence of ammonium persulfate as the terminal oxidant. The dehydrogenative nitration represents the first atom-economic example of construction of nitroaromatic olefins from arylethanes, which provides a new route toward a class of nitroaromatic olefins. A wide range of arylethanes including neutral ethylnaphthalenes, 1,1-diphenylethanes, phenylethanes and 4-ethyl-1,1'-biphenyl were suitable in this transformation, affording $E$-nitroaromatic olefins in moderate to good yields. However, ethylnaphthalenes bearing strong electron-donating group and the sterically hindered 1-isopropylnaphthalene were ineffective in this transformation. Ongoing work seeks to gain further insights into the mechanism of this reaction and to expand the reaction scope of other substrates.

\section{Experimental section}

\subsection{General methods}

All reactions were performed in a glass pressure tube equipped with a magnetic stir bar under argon unless otherwise noted. Flash column chromatography was performed using silica gel (6-nm pore size, $32 \sim 63 \mu \mathrm{m}$, standard 15 grade). Solvents and the other chemicals were bought from commercial sources and used as received unless otherwise noted.

Melting points were measured using a WC-1 microscopic apparatus and were uncorrected. GC-MS spectra were recorded on a Thermo Fisher Scientific DSQ II GC-MS spectrometer. HPLC yields were measured on a LC-MSD-Trap-XCT instrument. The high resolution mass spectrum was received via an Agilent Technologies 6540 UHD Accurate-mass Q-Tof LC/MS. NMR spectra were obtained on a Bruker DPX 400 systems using $\mathrm{CDCl}_{3}$ as solvent, TMS as internal standard substance, with proton and carbon resonances at 400 and $100 \mathrm{MHz}$, respectively.

\subsection{General procedure}

4.2.1 General procedure for $\mathrm{Cu} / \mathrm{Ag}$-cocatalyzed dehydrogenative nitration of 1-ethylnaphthalene with $\mathrm{Ba}-$ $\left(\mathrm{NO}_{3}\right)_{2}$

Firstly, in a dried sealed tube charged with a magnetic stirring bar, $\mathrm{CF}_{3} \mathrm{SO}_{3} \mathrm{H}$ (6 mmol) was dissolved in dry DMF $(0.5 \mathrm{~mL})$ at $0{ }^{\circ} \mathrm{C}$ under the protection of Ar. Then, the resulting solution was allowed to warm to room temperature. Next, $\mathrm{Ba}\left(\mathrm{NO}_{3}\right)_{2}(0.6 \mathrm{mmol}), \mathrm{CuOAc}(0.015 \mathrm{mmol})$, 4,7-diphenyl-1,10-phenathroline $(0.015 \mathrm{mmol}), \mathrm{K}_{3} \mathrm{PO}_{4}(0.3$ $\mathrm{mmol})$ and DMF $(0.5 \mathrm{~mL})$ were added into the reaction tube under a stream of argon. After stirring for a several minutes, $\left(\mathrm{NH}_{4}\right)_{2} \mathrm{~S}_{2} \mathrm{O}_{8}(0.45 \mathrm{mmol})$ and $\mathrm{CF}_{3} \mathrm{COOAg}(0.06$ mmol) were added, followed by the addition of 1-ethylnaphthalene $(0.3 \mathrm{mmol})$ in DMF $(1.0 \mathrm{~mL})$. The reaction mixture was evacuated by vacuum-argon cycles (3 times). The reaction was carried out under a sealed tube at $100{ }^{\circ} \mathrm{C}$ for $16 \mathrm{~h}$. After the reaction was complete, the mixture was washed with saturated sodium bicarbonate solution and extracted with ethyl acetate $(10 \mathrm{~mL} \times 3)$. The combined organic layer was dried with anhydrous $\mathrm{MgSO}_{4}$ and filtered. The filtrate was concentrated in vacuo. The crude product was purified by flash chromatography on silica gel using hexane/ethyl acetate $(V: V=10: 1)$ as the eluent to give the pure product.

\subsection{Characterization data of dehydronitration prod- ucts}

(E)-1-(2-Nitrovinyl)naphthalene (2a): Yellow solid (53 mg, yield 89\%). m.p. 83 $84{ }^{\circ} \mathrm{C}$ (lit. ${ }^{[63]} 82 \sim 84{ }^{\circ} \mathrm{C}$ ); ${ }^{1} \mathrm{H}$ NMR (400 MHz, $\left.\mathrm{CDCl}_{3}\right) \delta: 8.81(\mathrm{~d}, J=13.6 \mathrm{~Hz}, 1 \mathrm{H}), 8.11$ $(\mathrm{d}, J=8.4 \mathrm{~Hz}, 1 \mathrm{H}), 7.98(\mathrm{~d}, J=8.0 \mathrm{~Hz}, 1 \mathrm{H}), 7.89$ (d, $J=$ $8.0 \mathrm{~Hz}, 1 \mathrm{H}), 7.73(\mathrm{~d}, J=7.2 \mathrm{~Hz}, 1 \mathrm{H}), 7.64 \sim 7.48(\mathrm{~m}, 4 \mathrm{H})$; ${ }^{13} \mathrm{C} \mathrm{NMR}\left(100 \mathrm{MHz}, \mathrm{CDCl}_{3}\right) \delta: 138.5,136.1,133.8,132.6$, 131.6, 129.1, 127.8, 127.0, 126.8, 126.4, 125.4, 123.0; MS $\mathrm{m} / \mathrm{z}: 199.0[\mathrm{M}]^{+}$.

(E)-2-(2-Nitrovinyl)naphthalene (2b) Yellow solid (31 mg, yield 52\%). m.p. $128 \sim 130{ }^{\circ} \mathrm{C}$ (lit. ${ }^{[63]} 128 \sim 130{ }^{\circ} \mathrm{C}$ ); ${ }^{1} \mathrm{H}$ NMR (400 MHz, $\left.\mathrm{CDCl}_{3}\right) \delta: 8.16(\mathrm{~d}, J=13.6 \mathrm{~Hz}, 1 \mathrm{H})$, 
$8.02(\mathrm{~s}, 1 \mathrm{H}), 7.91 \sim 7.85(\mathrm{~m}, 3 \mathrm{H}), 7.70(\mathrm{~d}, J=13.6 \mathrm{~Hz}$, $1 \mathrm{H}), 7.61 \sim 7.56(\mathrm{~m}, 3 \mathrm{H}) ;{ }^{13} \mathrm{C} \mathrm{NMR}\left(100 \mathrm{MHz}, \mathrm{CDCl}_{3}\right) \delta$ : $139.2,137.2,134.9,133.2,132.3,129.4,128.8,128.4$, 128.0, 127.6, 127.3, 123.3; MS $m / z: 199.1[\mathrm{M}]^{+}$.

(E)-1-Methoxy-4-(2-nitro-1-phenylvinyl)benzene (2e): Yellow oil (64 mg, yield 84\%). ${ }^{64]}{ }^{1} \mathrm{H}$ NMR (400 MHz, $\left.\mathrm{CDCl}_{3}\right) \delta: 7.46 \sim 7.44(\mathrm{~m}, 1 \mathrm{H}), 7.40 \sim 7.35(\mathrm{~m}, 3 \mathrm{H})$, $7.30 \sim 7.26(\mathrm{~m}, 2 \mathrm{H}), 7.17(\mathrm{dd}, J=6.8,2.0 \mathrm{~Hz}, 2 \mathrm{H}), 6.93$ $(\mathrm{dd}, J=6.8,2.0 \mathrm{~Hz}, 2 \mathrm{H}) ;{ }^{13} \mathrm{C}$ NMR $\left(100 \mathrm{MHz}, \mathrm{CDCl}_{3}\right) \delta$ : $160.7,150.5,137.8,133.7,131.0,130.8,129.2,128.8$, 127.5, 113.9, 55.3; MS $m / z: 255.0[\mathrm{M}]^{+}$.

1-Methoxy-2-[1-(4-methoxyphenyl)-2-nitrovinyl]benzene (2f): Yellow oil (69 mg, yield 81\%). ${ }^{1} \mathrm{H}$ NMR (400 MHz, $\left.\mathrm{CDCl}_{3}\right) \delta: 7.42(\mathrm{~s}, 1 \mathrm{H}), 7.35 \sim 7.31(\mathrm{~m}, 1 \mathrm{H}), 7.19 \sim 7.17$ $(\mathrm{m}, 2 \mathrm{H}), 6.92 \sim 6.82(\mathrm{~m}, 3 \mathrm{H}), 6.80(\mathrm{dd}, J=6.8,2.0 \mathrm{~Hz}$, $2 \mathrm{H}), 3.75(\mathrm{~s}, 3 \mathrm{H}), 3.66(\mathrm{~s}, 3 \mathrm{H}) ;{ }^{13} \mathrm{C}$ NMR $(100 \mathrm{MHz}$, $\left.\mathrm{CDCl}_{3}\right) \delta: 161.9,156.7,146.6,134.0,130.5,130.2,129.9$, $129.1,125.1,120.6,114.3,111.0,55.7,55.4 ; \mathrm{MS} \mathrm{m} / \mathrm{z}$ : $284.9[\mathrm{M}]^{+}$; HRMS $\left(\mathrm{ESI}^{+}\right.$) calcd for $\mathrm{C}_{16} \mathrm{H}_{16} \mathrm{NO}_{4}[\mathrm{M}+\mathrm{H}]^{+}$ 286.1074, found 286.1072.

4,4'-(2-Nitroethene-1,1-diyl)bis(methylbenzene) (2g): Yellow solid (46 mg, yield 61\%). m.p. $108 \sim 110{ }^{\circ} \mathrm{C}$ (lit. $\left.{ }^{[65]} 110 \sim 111{ }^{\circ} \mathrm{C}\right) ;{ }^{1} \mathrm{H}$ NMR (400 MHz, $\left.\mathrm{CDCl}_{3}\right) \delta: 7.40$ $(\mathrm{s}, 1 \mathrm{H}), 7.26 \sim 7.18(\mathrm{~m}, 6 \mathrm{H}), 7.11(\mathrm{~d}, J=8.4 \mathrm{~Hz}, 2 \mathrm{H}), 2.42$ $(\mathrm{s}, 3 \mathrm{H}), 2.39(\mathrm{~s}, 3 \mathrm{H}) ;{ }^{13} \mathrm{C} \mathrm{NMR}\left(100 \mathrm{MHz}, \mathrm{CDCl}_{3}\right) \delta$ : $150.9,141.5,139.5,134.6,133.5,132.7,129.6,129.2$, 129.03, 128.98, 21.5, 21.4; MS m/z: $252.9[\mathrm{M}]^{+}$.

(E)-1-Methoxy-2-(2-nitro-1-phenylvinyl)benzene (2h): Yellow solid (37 mg, yield 48\%). m.p. $77 \sim 79{ }^{\circ} \mathrm{C} ;{ }^{1} \mathrm{H}$ NMR (400 MHz, $\left.\mathrm{CDCl}_{3}\right) \delta: 7.47(\mathrm{~s}, 1 \mathrm{H}), 7.43 \sim 7.29(\mathrm{~m}$, $6 \mathrm{H}), 7.00 \sim 6.96(\mathrm{~m}, 3 \mathrm{H}), 3.73(\mathrm{~s}, 3 \mathrm{H}) ;{ }^{13} \mathrm{C}$ NMR $(100$ $\left.\mathrm{MHz}, \mathrm{CDCl}_{3}\right) \delta: 156.7,146.6,137.1,135.5,130.7,130.6$, 130.0, 128.8, 128.5, 124.9, 120.7, 111.1, 55.6; MS m/z: $255.1[\mathrm{M}]^{+}$; HRMS $\left(\mathrm{ESI}^{+}\right)$calcd for $\mathrm{C}_{15} \mathrm{H}_{14} \mathrm{NO}_{3}[\mathrm{M}+\mathrm{H}]^{+}$ 256.0968, found 256.0965.

4,4'-(2-Nitroethene-1,1-diyl)bis(methoxybenzene) (2i): Yellow solid (37 mg, yield 43\%). m.p. $110 \sim 112{ }^{\circ} \mathrm{C}$ (lit. $\left.{ }^{[65]} 113 \sim 114{ }^{\circ} \mathrm{C}\right) ;{ }^{1} \mathrm{H} \mathrm{NMR}\left(400 \mathrm{MHz}, \mathrm{CDCl}_{3}\right) \delta: 7.36$ (s, 1H), $7.23(\mathrm{~d}, J=8.8 \mathrm{~Hz}, 2 \mathrm{H}), 7.16(\mathrm{~d}, J=8.8 \mathrm{~Hz}, 2 \mathrm{H})$, $6.93(\mathrm{~d}, J=8.8 \mathrm{~Hz}, 2 \mathrm{H}), 6.89$ (d, $J=8.8 \mathrm{~Hz}, 2 \mathrm{H}), 3.86$ (s, $3 \mathrm{H}), 3.84(\mathrm{~s}, 3 \mathrm{H}) ;{ }^{13} \mathrm{C} \mathrm{NMR}\left(100 \mathrm{MHz}, \mathrm{CDCl}_{3}\right) \delta: 162.0$, $160.7,150.8,132.4,131.0,130.9,129.9,127.8,114.3$, 113.8, 55.5, 55.3; MS m/z: $284.9[\mathrm{M}]^{+}$.

(Z)-1-Chloro-4-(1-(4-methoxyphenyl)-2-nitrovinyl)benz ene (2j): Yellow solid (36 mg, yield 42\%). m.p. 119 $121{ }^{\circ} \mathrm{C} ;{ }^{1} \mathrm{H}$ NMR (400 MHz, $\left.\mathrm{CDCl}_{3}\right) \delta: 7.44 \sim 7.40(\mathrm{~m}$, $3 \mathrm{H}), 7.21(\mathrm{~d}, J=9.2 \mathrm{~Hz}, 2 \mathrm{H}), 7.16(\mathrm{~d}, J=8.4 \mathrm{~Hz}, 2 \mathrm{H}), 6.90$ $(\mathrm{d}, J=8.8 \mathrm{~Hz}, 2 \mathrm{H}), 3.84(\mathrm{~s}, 3 \mathrm{H}) ;{ }^{13} \mathrm{C} \mathrm{NMR}(100 \mathrm{MHz}$, $\left.\mathrm{CDCl}_{3}\right) \delta: 162.3,149.5,135.4,134.2,133.1,130.6,130.3$, 128.8, 128.7, 114.5, 55.5; MS m/z: $288.8[\mathrm{M}]^{+}$; HRMS $\left(\mathrm{ESI}^{+}\right.$) calcd for $\mathrm{C}_{15} \mathrm{H}_{13} \mathrm{ClNO}_{3}[\mathrm{M}+\mathrm{H}]^{+}$290.0578, found 290.0577.

1-Chloro-4-(2-nitro-1-phenylvinyl)benzene (2k): Yellow solid (24 mg, yield 31\%). ${ }^{[64]}$ m.p. $66 \sim 69{ }^{\circ} \mathrm{C} ;{ }^{1} \mathrm{H}$ NMR $\left(400 \mathrm{MHz}, \mathrm{CDCl}_{3}\right) \delta: 7.43 \sim 7.35(\mathrm{~m}, 6 \mathrm{H}), 7.28 \sim$ $7.16(\mathrm{~m}, 4 \mathrm{H}) ;{ }^{13} \mathrm{C} \mathrm{NMR}\left(100 \mathrm{MHz}, \mathrm{CDCl}_{3}\right) \delta: 149.3$, $149.2,137.3,136.7,135.6,135.5,135.1,134.6,134.5$,
$133.9,131.2,130.3,130.1,129.6,129.2,129.0,128.89$, 128.86, 128.8, 128.7; MS m/z: $259.0[\mathrm{M}]^{+}$.

(2-Nitroethene-1,1-diyl)dibenzene (2l): ${ }^{[66]}$ Yellow solid (37 mg, yield 55\%). m.p. 82 84 ${ }^{\circ} \mathrm{C} ;{ }^{1} \mathrm{H}$ NMR (400 MHz, $\left.\mathrm{CDCl}_{3}\right) \delta: 7.45 \sim 7.37(\mathrm{~m}, 7 \mathrm{H}), 7.30 \sim 7.22(\mathrm{~m}, 4 \mathrm{H}) ;{ }^{13} \mathrm{C}$ NMR $\left(100 \mathrm{MHz}, \mathrm{CDCl}_{3}\right) \delta: 150.5,137.1,135.6,134.4$, $130.9,129.3,128.92,128.90,128.8,128.5$; MS $m / z: 225.1$ $[\mathrm{M}]^{+}$.

(E)-1-Methoxy-4-(2-nitrovinyl)benzene (2n): Yellow solid (40 mg, yield 74\%). m.p. 89 91 ${ }^{\circ} \mathrm{C}$ (lit. ${ }^{[66]} 87 \sim$ $\left.90{ }^{\circ} \mathrm{C}\right) ;{ }^{1} \mathrm{H}$ NMR $\left(400 \mathrm{MHz}, \mathrm{CDCl}_{3}\right) \delta: 7.97$ (d, $J=13.6$ $\mathrm{Hz}, 1 \mathrm{H}), 7.53 \sim 7.49(\mathrm{~m}, 3 \mathrm{H}), 6.95(\mathrm{~d}, J=8.8 \mathrm{~Hz}, 2 \mathrm{H})$, $3.87(\mathrm{~s}, 3 \mathrm{H}) ;{ }^{13} \mathrm{C}$ NMR $\left(100 \mathrm{MHz}, \mathrm{CDCl}_{3}\right) \delta: 163.0,139.0$, 135.1, 131.2, 122.6, 114.9, 55.5; MS m/z: $179.1[\mathrm{M}]^{+}$.

(E)-1-(2-Nitrovinyl)-4-(pentyloxy)benzene (2o): Yellow oil (32 mg, yield 45\%). ${ }^{1} \mathrm{H}$ NMR (400 $\left.\mathrm{MHz}, \mathrm{CDCl}_{3}\right) \delta$ : $7.93(\mathrm{~d}, J=13.2 \mathrm{~Hz}, 1 \mathrm{H}), 7.50 \sim 7.44(\mathrm{~m}, 3 \mathrm{H}), 6.90(\mathrm{dd}$, $J=6.8,2.0 \mathrm{~Hz}, 2 \mathrm{H}), 3.97(\mathrm{t}, J=6.4 \mathrm{~Hz}, 2 \mathrm{H}), 1.79 \sim 1.75$ $(\mathrm{m}, 2 \mathrm{H}), 1.41 \sim 1.34(\mathrm{~m}, 4 \mathrm{H}), 0.90(\mathrm{t}, J=5.4 \mathrm{~Hz}, 3 \mathrm{H}) ;{ }^{13} \mathrm{C}$ NMR $\left(100 \mathrm{MHz}, \mathrm{CDCl}_{3}\right) \delta: 162.6,139.1,134.9,131.2$, $122.3,115.4,68.4,28.8,28.1,22.4,14.0$; MS m/z: 235.1 $[\mathrm{M}]^{+}$; HRMS $\left(\mathrm{ESI}^{+}\right.$) calcd for $\mathrm{C}_{13} \mathrm{H}_{18} \mathrm{NO}_{3}[\mathrm{M}+\mathrm{H}]^{+}$ 236.1281, found 236.1280.

(E)-4-(2-Nitrovinyl)-1,1'-biphenyl (2p): Yellow solid (30 mg, yield 44\%). m.p. $192 \sim 194{ }^{\circ} \mathrm{C}$ (lit. ${ }^{[67]} 190 \sim$ $\left.192{ }^{\circ} \mathrm{C}\right) ;{ }^{1} \mathrm{H}$ NMR $\left(400 \mathrm{MHz}, \mathrm{CDCl}_{3}\right) \delta: 8.02(\mathrm{~d}, J=13.6$ $\mathrm{Hz}, 1 \mathrm{H}), 7.67 \sim 7.58(\mathrm{~m}, 7 \mathrm{H}), 7.47 \sim 7.38(\mathrm{~m}, 3 \mathrm{H}) ;{ }^{13} \mathrm{C}$ NMR $\left(100 \mathrm{MHz}, \mathrm{CDCl}_{3}\right) \delta: 145.0,139.6,138.7,136.9$, $129.7,129.1,128.9,128.4,128.0,127.1$; MS m/z: 225.1 $[\mathrm{M}]^{+}$.

Supporting Information Copies of ${ }^{1} \mathrm{H}$ NMR and ${ }^{13} \mathrm{C}$ NMR spectra of the dehydronitration products. The Supporting Information is available free of charge via the Internet at http://sioc-journal.cn/.

\section{References}

[1] Reddy, M. A.; Jain, N.; Yada, D.; Kishore, C.; Reddy, V. J.; Reddy, P. S.; Addlagatta, A.; Kalivendi, S. V.; Sreedhar, B. J. Med. Chem. 2011, 54, 6751.

[2] Lu, L. Q.; Chen, J. R.; Xiao, W. J. Acc. Chem. Res. 2012, 45, 1278.

[3] Kaap, S.; Quentin, I.; Tamiru, D.; Shaheen, M.; Eger, K.; Steinfelder, H. J. Biochem. Pharmacol. 2003, 65, 603.

[4] Uehara, H.; Imashiro, R.; Hernández-Torres, G.; Barbas III, C. F. Proc. Natl. Acad. Sci. U. S. A. 2010, 107, 20672.

[5] Meah, Y.; Massey, V. Proc. Natl. Acad. Sci. U. S. A. 2000, 97, 10733.

[6] Ishii, T.; Fujioka, S.; Sekiguchi, Y.; Kotsuki, H. J. Am. Chem. Soc. 2004, 126, 9558 .

[7] Huang, H. B.; Jacobsen, E. N. J. Am. Chem. Soc. 2006, 128, 7170.

[8] Tripathi, C. B.; Kayal, S.; Mukherjee, S. Org. Lett. 2012, 14, 3296.

[9] Bai, B.; Wang, L.; Yang, J.; Cai, L. L.; Liu, Q. J.; Xi, Gao. L.; Zhao, Z. W.; Mao, D. B.; Chen, Z. F. Chin. J. Org. Chem. 2019, 39, 1053 (in Chinese).

(白冰, 王龙, 杨静, 蔡莉莉, 刘前进, 席高磊, 赵志伟, 毛多斌, 陈芝飞, 有机化学, 2019, 39, 1053.)

[10] Luo, S. P.; Wang, L. P.; Yue, H. D.; Le, Z. G.; Yang, W. L.; Xu, D. Q.; Xu, Z. Y. Acta Chim. Sinica 2006, 64, 1483.

[11] Evans, D. A.; Mito, S.; Seidel, D. J. Am. Chem. Soc. 2007, 129, 11583 . 
[12] March, J. Advanced Organic Chemistry, 3rd ed., John Wiley \& Sons, New York, 1985.

[13] Albrecht, L.; Dickmeiss, G.; Acosta, F. C.; Rodrí guez-Escrich, C.; Davis, R. L.; Jorgensen, K. A. J. Am. Chem. Soc. 2012, 134, 2543.

[14] Liu, Y. K.; Nappi, M.; Arceo, E.; Vera, S.; Melchiorre, P. J. Am. Chem. Soc. 2011, 133, 15212.

[15] Arai, T.; Mishiro, A.; Yokoyama, N.; Suzuki, K.; Sato, H. J. Am. Chem. Soc. 2010, 132, 5338.

[16] Denmark, S. E.; Thorarensen, A. Chem. Rev. 1996, 96, 137.

[17] Yan, L. J.; Xu, H.; Wang, Y.; Dong, J. W.; Wang, Y. C. Chin. J. Org. Chem. 2020, 40, 284 (in Chinese). (严丽君, 徐菡, 王艳, 董建伟, 王永超, 有机化学, 2020, 40, 284.)

[18] Basavaiah, D.; Reddy, B. S.; Badsara, S. S. Chem. Rev. 2010, 110, 5447.

[19] Nair, D. K.; Mobin, S. M.; Namboothiri, I. N. N. Org. Lett. 2012, $14,4580$.

[20] Quan, X. J.; Ren, Z. H.; Wang, Y. Y.; Guan, Z. H. Org. Lett. 2014, $16,5728$.

[21] Chen, Y. F.; Nie, G.; Zhang, Q.; Ma, S.; Li, H.; Hu, Q. Q. Org. Lett. 2015, 17, 1118 .

[22] Kurti, L.; Czako, B. Strategic Applications of Named Reactions in Organic Synthesis, Elsevier Academic Press, London, 2005.

[23] Fioravanti, S.; Pellacani, L.; Tardella, P. A.; Vergari, M. C. Org. Lett. 2008, 10, 1449.

[24] Hassner, A.; Kropp, J. E.; Kent, G. J. J. Org. Chem. 1969, 34, 2628.

[25] Ranganathan, S.; Kar, S. K. J. Org. Chem. 1970, 35, 3962.

[26] Corey, E. J.; Estreicher, H. J. Am. Chem. Soc. 1978, 100, 6294.

[27] Barluenga, J.; Rodríguez, M. A.; Campos, P. J.; Asensio, G. J. Chem. Soc., Chem. Commun. 1987, 1491.

[28] Barluenga, J.; Rodríguez, M. A.; Campos, P. J. J. Chem. Soc., Perkin Trans. 1 1990, 2807.

[29] Sy, W. W.; By, A. W. Tetrahedron Lett. 1985, 26, 1193.

[30] Jew, S. S.; Kim, H. D.; Cho, Y. S.; Cook, C. H. Chem. Lett. 1986, $15,1747$.

[31] Hwu, J. R.; Chen, K. L.; Ananthan, S.; Patel, H. V. Organometallics 1996, 15,499

[32] Ghosh, D.; Nichols, D. E. Synthesis 1996, 195.

[33] Suzuki, H.; Mori, T. J. Org. Chem. 1997, 62, 6498.

[34] Mukaiyama, T.; Hata, E.; Yamada, T. Chem. Lett. 1995, 24, 505.

[35] Hata, E.; Yamada, T.; Mukaiyama, T. Bull. Chem. Soc. Jpn. 1995, $68,3629$.

[36] Jovel, I.; Prateeptongkum, S.; Jackstell, R.; Vogl, N.; Weckbecker, C.; Beller, M. Adv. Synth. Catal. 2008, 350, 2493.

[37] Varma, R. S.; Naicker, K. P.; Liesent, P. J. Tetrahedron Lett. 1998, 39, 3977.

[38] Manna, S.; Jana, S.; Saboo, T.; Maji, A.; Maiti, D. Chem. Commun. 2013, 49, 5286

[39] Rokade, B. V.; Prabhu, K. R. Org. Biomol. Chem. 2013, 11, 6713.

[40] Das, J. P.; Sinha, P.; Roy, S. Org. Lett. 2002, 4, 3055.
[41] Baruah, D.; Pahari, P.; Konwar, D. Tetrahedron Lett. 2015, 56, 2418.

[42] Yang, Z.; Li, J.; Hua, J.; Yang, T.; Yi, J. M.; Zhou, C. S. Synlett 2017, 28, 1079.

[43] Luo, Z. G.; Xu, F.; Fang, Y. Y.; Liu, P.; Xu, X. M.; Feng, C. T.; Li, Z.; He, J. Res. Chem. Intermed. 2016, 42, 6079.

[44] Maity, S.; Manna, S.; Rana, S.; Naveen, T.; Mallick, A.; Maiti, D. J. Am. Chem. Soc. 2013, 135, 3355.

[45] Naveen, T.; Maity, S.; Sharma, U.; Maiti, D. J. Org. Chem. 2013, $78,5949$.

[46] Maity, S.; Naveen, T.; Sharma, U.; Maiti, D. Org. Lett. 2013, 15, 3384.

[47] Zhao, A.; Jiang, Q.; Jia, J.; Xu, B.; Liu, Y. F.; Zhang, M. Z.; Liu, Q.; Luo, W. P.; Guo, C. C. Tetrahedron Lett. 2016, 57, 80.

[48] Whiting, K.; Carmona, L. G.; Sousa, T. Renewable Sustainable Energy Rev. 2017, 76, 202.

[49] Degnan, T. F. J. Catal. 2003, 216, 32.

[50] Grant, J. T.; Venegas, J. M.; McDermott, W. P.; Hermans, I. Chem. Rev. 2018, 118, 2769.

[51] Kumar, A.; Bhatti, T. M.; Goldman, A. S. Chem. Rev. 2017, 117, 12357.

[52] Wang, Y. L.; Qian, L.; Huang, Z. D.; Liu, G. X.; Huang, Z. Chin. J. Chem. 2020, 38, 837.

[53] Sathyamoorthi, S.; Banerjee, S. ChemistrySelect 2017, 2, 10678

[54] Sathyamoorthi, S.; Du Bois, J. Org. Lett. 2016, 18, 6308.

[55] Banerjee, S.; Sathyamoorthi, S.; Du Bois, J.; Zare, R. N. Chem. Sci. 2017, 8, 7003 .

[56] Manna, S.; Antonchick, A. P. Chem.-Eur. J. 2017, 23, 7825.

[57] Burkhard, C. A.; Brown, J. F., Jr. US 2867669, 1959.

[58] Tang, X. J.; Dolbier, Jr. W. R. Angew. Chem. Int. Ed. 2015, 54, 4246.

[59] Ambala, S.; Singh, R.; Singh, M.; Cham, P. S.; Gupta, R.; Munagala, G.; Yempalla, K. R.; Vishwakarma, R. A.; Singh, P. P. RSC Adv. 2019, 9, 30428.

[60] Huie, R. E.; Clifton, C. L.; Kafafi, S. A. J. Phys. Chem. 1991, 95 , 9336.

[61] Huie, R. E.; Clifton, C. L. J. Phys. Chem. 1990, 94, 8561.

[62] Liu, Y.; Wang, Q. L.; Chen, Z.; Zhou, Q.; Zhou, C. S.; Xiong, B. Q.; Zhang, P. L. ; Yang, C. A.; Tang, K. W. Org. Biomol. Chem. 2019, $17,1365$.

[63] Manna, S.; Jana, S.; Saboo, T.; Maji, A.; Maiti, D. Chem. Commun. 2013, 49, 5286.

[64] Gross, Z.; Hoz, S. J. Am. Chem. Soc. 1988, 110, 7489.

[65] Hsieh, T. H. H.; Dong, V. M. Tetrahedron 2009, 65, 3062.

[66] Zhao, A.; Jiang, Q.; Jia, J.; Xu, Bin.; Liu, Y. F.; Zhang, M. Z.; Liu, Q.; Luo, W. P.; Guo, C. C. Tetrahedron Lett. 2016, 57, 80.

[67] Ambala, S.; Singh, R.; Singh, M.; Cham, P. S.; Gupta, R.; Munagala, G.; Yempalla, K. R.; Vishwakarma, R. A.; Singh, P. P. RSC Adv. 2019, 9, 30428. 